multiple partners). 194 (50\%) had recently used recreational drugs (within 3 months; 34\% "Chemsex" substances). 157 (41\%) reported a recent STI (6 months). 223 (58\%) reported that they strongly believed they would benefit from PrEP. However, 42/223 (19\%) reported no condomless sex. Concerns around taking PrEP were cited by 76 (20\%). 167 (43\%) expressed a preference for daily PrEP; 139 (38\%) for coitallydriven. 311 (80\%) supported PrEP delivery by sexual health clinics to MSM, and $233(60 \%)$ to any-one who requests it. 112 (29\%) agreed a prescription charge was appropriate. 17 respondents (4\%) reported having already taken PrEP: 35\% using medication acquired as PEP, and 30\% acquiring PrEP privately. 7/17 (41\%) reported decreased condom since commencing PrEP.

Discussion/conclusion This comprehensive questionnaire study demonstrates a high willingness to use PrEP in a cohort of atrisk MSM. These data should inform the commissioning process of this efficacious biological intervention.

\section{ESTABLISHMENT OF A MONITORING SERVICE FOR MEN WHO HAVE SEX WITH MEN (MSM) TAKING GENERIC CO-FORMULATED TENOFOVIR DISOPROXIL FUMARATE (TDF)/EMTRICITABINE (FTC) AS PRE-EXPOSURE PROPHYLAXIS (PREP) AGAINST HIV INFECTION}

${ }^{1}$ Nneka Nwokolo, ${ }^{2}$ Xinzhu Wang, ${ }^{1,2}$ Marta Boffito, ${ }^{2}$ Myra McClure, ${ }^{1}$ Tara Suchak* ${ }^{1}$ Gary Whitlock. '56 Dean Street, Chelsea \& Westminster Hospital, London, UK; ${ }^{2}$ Imperial College, London, UK

10.1136/sextrans-2016-052718.14

Background/introduction Truvada ${ }^{\circledR}$ (TDF/FTC) PrEP taken daily or intermittently reduces HIV acquisition by over $86 \%$. However, PrEP is only available privately in the UK, costing upwards of $£ 400$ for 30 tablets. Online generic TDF/FTC is significantly cheaper at $£ 35$ - $£ 50$ for 30 tablets. There are, however, authenticity concerns about online medicines. Additionally, HIV infection should be excluded in individuals taking PrEP and baseline assessments of hepatitis B and renal function performed which may not occur with online PrEP.

Since February 2016, we have provided assessment and therapeutic drug monitoring to individuals on generic TDF/FTC to ensure safety and medication integrity.

Aim(s)/objectives To review characteristics of individuals taking generic TDF/FTC.

Methods Service evaluation of individuals taking generic TDF/ FTC attending a London sexual health service. Data on the first 44 patients were collected: demographics, HIV and renal function testing, hepatitis B status, baseline STIs, regimen, source of PrEP.

Results All MSM; mean age 41 years (28-73); 77\% White; 33/ $44(75 \%)$ on PrEP at time of attendance; all HIV antibody negative prior to commencement. Mean eGFR $81.5 \mathrm{ml} / \mathrm{min}, 65 \%$ had documented hepatitis B immunity. One STI (syphilis) was identified at baseline. 93\% were taking daily PrEP and $86 \%$ obtained Cipla manufactured Tenvir-EM ${ }^{\circledR}$ from United Pharmacies. Tenofovir and FTC levels were measured in 18/44 (41\%), all results demonstrating presence of adequate active compound. Discussion/conclusion Numbers of individuals requiring monitoring on generic TDF/FTC are increasing. It is reassuring that so far, drug levels suggest appropriate quantities of tenofovir and FTC in Tenvir-EM ${ }^{\circledR}$; however, more data are needed.

\section{RENAL FUNCTION AT BASELINE AND MONTH 1 IN THE PROUD STUDY, A PRAGMATIC OPEN LABEL RANDOMISED TRIAL OF TRUVADA AS PRE-EXPOSURE PROPHYLAXIS}

${ }^{1}$ lain Reeves*, ${ }^{2}$ Ellen White, ${ }^{2}$ Elizabeth Brodnicki, ${ }^{2}$ David Dunn, ${ }^{2}$ Sheena McCormack, ${ }^{3}$ Tristan Barber, ${ }^{3}$ Ann Sullivan, ${ }^{5}$ Charles Lacey, ${ }^{4}$ Hannah Alexander. ${ }^{1}$ Homerton University Hospital, London, UK; ${ }^{2}$ MRC CTU, London, UK; ${ }^{3}$ Chelsea and Westminster NHS Trust, London, UK; ${ }^{4}$ Kings College Hospital, London, UK; ${ }^{5}$ Hull York Medical School, York, UK

\subsection{6/sextrans-2016-052718.15}

Background/introduction Quarterly monitoring of creatinine is likely to be recommended by WHO for those on PrEP, even though there were no significant differences in creatinine in placebo-controlled trials. Establishing the appropriate level of monitoring of PrEP is important.

Methods PROUD is an open-label, randomised trial of Truvada as PrEP in MSM. HIV serology and serum creatinine was done at PrEP baseline ('start'). Clinics were advised to collect creatinine or urinary protein-creatinine ratio (UPCR) if there was $\geq 1+$ protein on urinalysis at the month 1 visit (m1). Here we present the renal monitoring results at "start" and $\mathrm{m} 1$ with eGFR $\left(\mathrm{ml} / \mathrm{min} / 1.73 \mathrm{~m}^{2}\right)$ calculated by the CKD-EPI equation.

Results 445 (93\%) of 481 had baseline creatinine, 13 (3\%) had UPCR, and $23(5 \%)$ neither. The median eGFR was 106. Only one was $<60($ eGFR $=49)$, probably due to dietary creatinine supplementation. 260 (59\%) of 443 had a $\mathrm{m} 1$ creatinine, creating 246 paired results. On average, eGFR was 1.50 lower at $\mathrm{m} 1$. Seven (4\%) of 194 with eGFR >90 dropped 20\%, one to 59. He stopped PrEP and did not attend thereafter. Of the 7 , none had abnormal urinalysis; 4 had UPCR - all normal. 41 (79\%) of 52 with eGFR 60-90 at baseline remained at this level, the remainder increased to $>90$.

Discussion/conclusion The mean change in eGFR at month 1 is not clinically significant. Excepting one individual who could not be further evaluated, there were no clinically meaningful changes at $\mathrm{m} 1$. Further work will explore the relationships between eGFR and proteinuria.

\section{CHEMSEX RELATED ADMISSIONS TO A CITY CENTRE HOSPITAL}

${ }^{1}$ Chris Ward*, 'Debbie Thomas, ${ }^{2,1}$ Terri Anderson, ${ }^{1}$ Rebecca Evans, ${ }^{1}$ Orla McQuillan. ${ }^{1}$ Central Manchester University Hospitals NHS Foundation Trust, Manchester, UK; ${ }^{2}$ Addiction Dependency Solutions, Manchester, UK

\subsection{6/sextrans-2016-052718.16}

Background/introduction Recreational drug use (RDU), particularly the chemsex drugs mephedrone, crystal methamphetamine and gamma-hydroxybutyric acid (GHB) are associated with significant harms. Occasionally this has led to hospital admission with significant morbidity and mortality.

Aim(s)/objectives To review inpatient admissions from a large HIV service and look at RDU associations.

Methods A prospective analysis of admissions to an HIV inpatient service between April 2015 and March 2016 was conducted. Information was collected on demographics, admission details, complications and drug use.

Results From 194 admissions there were 19 (9.8\%) related to RDU. Median age was 33.5 (range 23-65). All were male and $18(94.7 \%)$ were men who have sex with men (MSM). 4 (21.1\%) were Hepatitis C co-infected. 5 (26.3\%) patients took 